PHYSICAL REVIEW B 96, 039907(E) (2017)

\title{
Erratum: Magnetotransport in a periodically modulated graphene monolayer [Phys. Rev. B 81, 085402 (2010)]
}

R. Nasir, K. Sabeeh, and M. Tahir

(Received 27 May 2017; published 31 July 2017)

DOI: 10.1103/PhysRevB.96.039907

There is a typographical error on the right-hand side of Eq. (15). Instead of "0" on the right-hand side of Eq. (15), the correct result is " $n$."

This correction does not effect the results, nor does it require a modification of the discussion. 